\title{
Correlation Analysis of Simultaneously Collected Bistatic and Monostatic Sea Clutter
}

\author{
Riccardo Palamà, Matthew \\ Ritchie, Hugh Griffiths, William \\ Miceli \\ Electronic and Electrical \\ Engineering Department \\ University College London \\ London, UK \\ r.palama@ucl.ac.uk
}

\author{
Francesco Fioranelli \\ School of Engineering \\ University of Glasgow \\ Glasgow, UK
}

\author{
Stephan Sandenbergh, Michael \\ Inggs \\ Department of Electrical \\ Engineering \\ University of Cape Town \\ Cape Town, South Africa
}

\begin{abstract}
In this work we analyse the correlation properties of simultaneous bistatic and monostatic polarimetric sea clutter data, collected by the NetRAD multistatic radar system. In particular, we study the temporal autocorrelation and crosscorrelation functions of the texture and speckle samples, as a function of the system geometry and of the polarization of the transmitter and receiver antennas. These features can vary significantly as a function of bistatic angle and the goal of this paper is to quantify these variations and extract any trends that are observed.
\end{abstract}

Keywords - bistatic radar, multistatic radar, sea clutter, correlation analysis

\section{INTRODUCTION}

Multistatic radar systems have been a topic of growing interest in the radar community for a number of years. These systems employ multiple transmitter and receiver nodes, in order to take advantage from the diversity, in both target and clutter returns, which is obtained by varying the system geometry. This can offer potential advantages over conventional monostatic radar, such as enhanced target signatures, improved detection using multi-perspective views, and the fact that the receive-only passive nodes are less complex and more compact, hence suitable to be mounted on UAVs or other unmanned platforms for enhanced surveillance operations [1],[2]. Fundamentally a multistatic sensor network has more information than a single monostatic sensor on the target and background scene, which if used optimally can provide significant gains in detection and tracking performance. In order to use this additional information, multistatic detection algorithms require effective modelling of the sea clutter statistics that are observed over the range of multistatic geometries utilized. This improved knowledge of clutter statistics and enhanced detection algorithms can be particularly beneficial for the identification, tracking, and classification of small maritime targets such as small boats, inflatables, and submarine periscopes.

There are very limited quantities of bistatic and multistatic sea clutter data available, as the required systems and measurements to collect them are complex and often related to classified research [1]. In this paper we aim to discuss the analysis of a dataset of bistatic sea clutter measurements collected in an experimental campaign in 2011. In particular, we investigate the temporal correlation properties of the sea clutter data which were collected by the NetRAD radar nodes, in both bistatic and monostatic configurations. The sea clutter samples consist of two fundamental components, i.e. a longlasting texture component, and a short-lasting speckle component. The autocorrelation properties of the texture and speckle carry important information about the level of nonstationarity of the clutter data. Typical values of the texture decorrelation time are of the order of seconds, whereas the speckle decorrelation time is of the order of milliseconds [3][4]. This is important in order to properly set the value of the coherent processing interval (CPI). In general, the temporal autocorrelation of sea clutter has been shown to affect radar detection performance, as the relation between the clutter-speckle and target decorrelation times impacts on the detection performance [5]. If the target signal decorrelates slower than the clutter speckle, the CPI value can be set close to the target decorrelation time, thus extracting greater target information from the received signal and increasing detection performance.

When applying multistatic detection algorithms for targets embedded in non-Gaussian clutter it is typically assumed that the clutter samples are statistically independent across different radar channels [6]. However, if - in real data such as those investigated here - this hypothesis is not verified, the performance of such multistatic detection techniques could be significantly reduced. In [6] the authors address an empirical study about the difference in detection performance between multichannel data - generated as statistically independent across channels - and real multistatic data. Results showed that the difference in detection performance between singlechannel and multichannel detectors is higher for syntheticallygenerated data, which is possibly due to a nonzero crosscorrelation between the radar channels. In [8] the authors proposed a multichannel detection algorithm, accounting for both the temporal autocorrelation of a single channel and the cross-correlation between two channels, but in the case of Gaussian disturbance.

In this work we analyse the cross-correlation between the bistatic and monostatic clutter data, for both the texture and speckle components. The level of cross-correlation present in 
the data relates directly to how valid the hypothesis assumption is for these detection algorithms. In addition, the analysis presented in this work aims at providing an insight on how the cross-correlation can be modelled to synthetically generate correlated multichannel data, whose statistical distribution is compound-Gaussian. The texture and speckle components of the sea clutter will be first separated, by estimating the texture through a block-averaging technique [3], then autocorrelation and cross-correlation functions will be measured for both the components. The behaviour of the texture and speckle autocorrelation will be studied as a function of the bistatic geometry, by extracting the values of the decorrelation time. On the other hand, the crosscorrelation information will be quantified by its maximum value for each bistatic geometry considered.

The paper is organized as follows. Section II presents the radar system and describe the experimental setup for data collection. Section III discusses the analysis of the texture and speckle, highlighting that the differences between the monostatic and bistatic datasets. Finally, section IV concludes the paper.

\section{SYSTEM AND DATASET DESCRIPTION}

The data analysed in this paper were collected using the multistatic S-band radar NetRAD, which uses three distinct but essentially identical radar nodes. The system was developed at University College London (UCL) over the past +10 years, and then upgraded in collaboration with the University of Cape Town in order to collect sea clutter data over baselines in the order of kilometers [9][10]. The radar parameters for the series of data analyzed here were $2.4 \mathrm{GHz}$ frequency, $45 \mathrm{MHz}$ linear up-chirp bandwidth, $1 \mathrm{kHz}$ Pulse Repetition Frequency (PRF), 130,000 pulses equal to 130 seconds per recording, and pulse length between $1.8 \mu$ s and 3 $\mu \mathrm{s}$, depending on the geometry. Two of the three NetRAD nodes were used for this data collection. One high power amplifier was used at one radar node to achieve a transmitting power of $450 \mathrm{~W}$, whereas a second node was used as passive receiver. The antennas had approximately $10^{\circ} \times 10^{\circ}$ beamwidth and $24 \mathrm{dBi}$ gain.

The data were collected on June 7th 2011 in South Africa at Misty Cliffs near the village of Scarborough and facing the open Atlantic Ocean on the west side of the Cape Peninsula. The baseline between the nodes was approximately $1830 \mathrm{~m}$, with a resulting grazing angle with respect to the sea surface varying within $0.7^{\circ}$ and $1.12^{\circ}$, so fairly low grazing angles. The bistatic angles were varied between $60^{\circ}$ and $120^{\circ}$ by manually rotating the antennas to point to a common clutter patch. This was done using optical telescopic sights and vernier bevel with mechanical azimuth readouts, providing a precision capability of $0.2^{\circ}$, roughly equal to $1 / 50^{\text {th }}$ of the beam-width. The values of bistatic angles considered were $60^{\circ}, 75^{\circ}, 85^{\circ}, 90^{\circ}, 95^{\circ}, 105^{\circ}$, and $120^{\circ}$. All the possible polarization combinations were collected, namely $\mathrm{VV}, \mathrm{HV}$, $\mathrm{HH}$, and $\mathrm{VH}$, although not simultaneously as the antennas needed to be rotated manually. However, the data collection at different bistatic angles for a given polarization was done almost simultaneously by changing the antennas azimuth.
Considering 7 bistatic angles and 4 polarization combinations, 28 sea clutter recordings were collected as a whole. The available wind data for the day reported wind speed of about $9 \mathrm{~m} / \mathrm{s}$ blowing from a South-Eastern direction, and wave height of between 1 and $3 \mathrm{~m}$ depending on the exact time of the day. It should be noted that the same location on the Scarborough area was also used for different data collection performed at different times and described in previous works [9][11][12]. Furthermore, in this work we report the results associated only to the range cell at the center of the intersection between the monostatic and bistatic antenna patterns [11].

\section{TEXTURE-SPECKLE ANALYSIS}

Sea clutter samples are usually modeled as compound Gaussian (CG) random variables. A complex CG random variable $\mathrm{W}$ is given by the product $w=\sqrt{\tau} x$, where the texture $\tau$ is real-valued and non-negative and the speckle $x$ is complex.

Due to their nature, texture and speckle have different autocorrelation properties. The texture indicates the local power of the sea clutter and is a long-lasting component, whereas the speckle is short-lasting. Hence, the texture decorrelation time $t_{\tau}$ is much longer than the speckle decorrelation time $t_{\mathrm{x}}$. The value of $t_{\tau}$ is typically of the order of seconds, whereas $t_{\mathrm{x}}$ is of the order of milliseconds, even if both quantities are influenced by the radar parameters - such as the carrier frequency, the pulse repetition frequency, the pulse length and the radar resolution - and by the environmental conditions (such as sea state and wave direction). The autocorrelation functions of the texture $R_{\tau}(m)$ - , speckle - $R_{x}(m)$ - and global clutter samples $R_{w}(m)$ - are related by,

$$
\begin{aligned}
R_{w}(m) & =E\left\{w(n) w^{*}(n+m)\right\} \\
& =E\left\{\sqrt{\tau(n)} \sqrt{\tau(n+m)} x(n) x^{*}(n+m)\right\} \\
& =E\{\sqrt{\tau(n)} \sqrt{\tau(n+m)}\} E\left\{x(n) x^{*}(n+m)\right\} \\
& =R_{\tau}(m) R_{x}(m)
\end{aligned}
$$

The speckle autocorrelation depends on the autocorrelation and cross-correlation functions of its in-phase and quadrature components $x_{\mathrm{I}}$ and $x_{\mathrm{Q}}$, i.e.

$$
\begin{aligned}
& R_{x}(m)=E\left\{x(n) x^{*}(n+m)\right\} \\
& \quad=E\left\{\left[x_{I}(n)+j x_{Q}(n)\right]\left[x_{I}(n+m)-j x_{Q}(n+m)\right]\right\} \\
& \quad=2\left[R_{I}(m)+j R_{I Q}(m)\right]
\end{aligned}
$$

where the autocorrelation functions of the in-phase - $R_{I}(m)$ and quadrature $-R_{Q}(m)$ - components are assumed to be identical [3], and $R_{I Q}(m)$ represents the cross-correlation function between the I and Q speckle samples, i.e. the imaginary part of $R_{x}(m)$. A consideration that arises from (1) is that the global autocorrelation function will match the autocorrelation function of the faster component, i.e. the 
speckle. Hence, the global decorrelation time is well approximated by the speckle decorrelation time.

In order to examine in detail the texture and speckle of sea clutter data, these components must be separated, which can be obtained by estimating the texture values. A simple estimation texture estimation technique [3] consists of averaging the squared absolute values of the clutter time samples (for a fixed range cell) over a time interval $T_{\mathrm{L}}$, i.e. over bursts of $L$ samples, where $L=T_{\mathrm{L}} \cdot P R F$,

$$
\hat{\tau}(k)=\frac{1}{L} \sum_{n=1+(k-1) L / 2}^{(k+1) L / 2}|x(n)|^{2} \quad k=1, \ldots, N_{b}
$$

where $N_{b}$ is the total number of bursts, $N_{b}=N_{p} / L$. In our analyses, the block dimension was set as 256 , which is 0.256 sec and has been selected from empirical evaluations of the texture estimation accuracy.

\section{A. Texture Autocorrelation}

After a method to estimate the texture values has been validated, it is possible to examine the statistical properties of the texture and speckle. The analysis of the autocorrelation among the temporal samples of the texture can give an indication of its degree of non-stationarity.

In particular, the autocorrelation coefficient was chosen as a representative parameter for this analysis, as its behaviour as a function of the time lag describes the degree of correlation between the samples of a stochastic process. The texture covariance was estimated as [3]

$$
R_{\tau}(l)=\frac{1}{N_{b}} \sum_{k=1}^{N_{b}-|l|}\left[\hat{\tau}(k)-\hat{\mu}_{\tau}\right]\left[\hat{\tau}(k+|l|)-\hat{\mu}_{\tau}\right]
$$

where $N_{b}$ is the number of texture samples and $\hat{\mu}_{\tau}$ is the texture sample mean. The texture correlation coefficient was computed as $\hat{\rho}_{\tau}(l)=R_{\tau}(l) / R_{\tau}(0)$.

Fig.1 shows the behaviour of the texture autocorrelation coefficient as a function of the time lag (in seconds), for the bistatic and monostatic data collected by NetRAD with a bistatic angle of $75^{\circ}$, both at horizontal and vertical polarization. In this figure, the bistatic $\mathrm{HH}$ texture seems to decorrelate more rapidly than the monostatic one, while the opposite appears true for the VV data. Furthermore, autocorrelation functions in Fig. 1 show some periodicities, i.e. smaller peaks due to the periodical motion of the sea waves. A smaller peak occurs at 9 seconds for bistatic VV data, at 13 seconds for monostatic VV.

It is usually assumed that the decorrelation time of a signal $\left(t_{\tau}\right.$ for the texture) is such that $\rho_{\tau}\left(t_{\tau}\right) \approx \exp (-1)$. In the case of co-polarized data (Fig.2a), horizontally polarized bistatic texture shows the lowest values of the decorrelation time, for bistatic angles smaller than $90^{\circ}$, and, in general, VV data show higher values of $t_{\tau}$ than $\mathrm{HH}$ ones. In the case of crosspolarized data, bistatic texture shows always higher values of $\mathrm{t}_{\tau}$ with respect to monostatic one, with a peak between $85^{\circ}$ $90^{\circ}$.

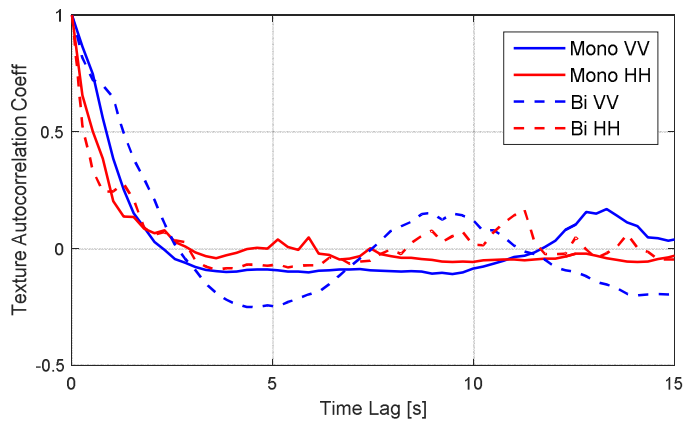

Fig. 1 - Texture autocorrelation coefficient as a function of the time lag (copolarized data, $\beta=75^{\circ}$ )

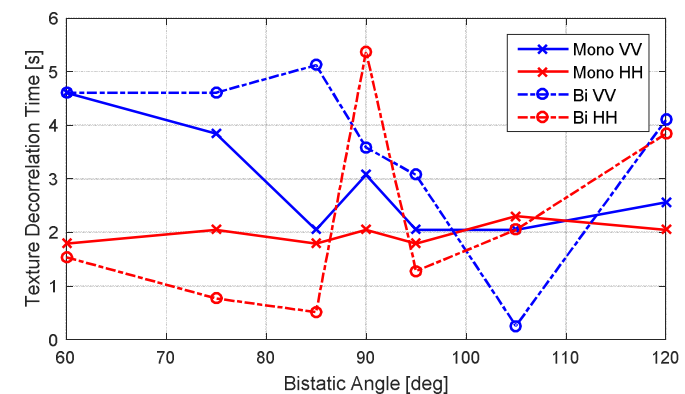

(a)

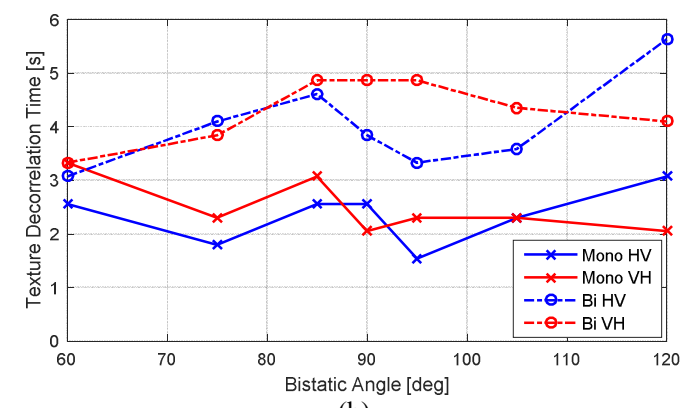

(b)

Fig. 2: Texture decorrelation times of (a) Co-polarized data, (b) Crosspolarized data

\section{B. Speckle Autocorrelation}

The temporal correlation of the speckle samples can by analysed by evaluating the values of the autocorrelation coefficient of its in-phase $\left(\rho_{I}\right)$ and quadrature $\left(\rho_{Q}\right)$ components and of their cross-correlation coefficient $\left(\rho_{I Q}\right)$. The temporal auto-covariance of the in-phase speckle component is given by [3]

$$
R_{I}(m)=\frac{1}{2 N_{b} L} \sum_{k=1}^{N_{b}} \frac{1}{\tau_{k}} \operatorname{Re}\left\{\sum_{n=1}^{N-1-|m|} x_{k}(n) x_{k}^{*}(n+m)\right\}
$$

whereas the cross-correlation of the I and Q components is

$R_{I Q}(m)=\frac{1}{2 N_{b} L} \sum_{k=1}^{N_{b}} \frac{1}{\tau_{k}} \operatorname{Im}\left\{\sum_{n=1}^{N-1-|m|} x_{k}(n) x_{k}^{*}(n+m)\right\}$

where $x_{k}(i)=x((k-1) L+i)$.

It should be noted that $\rho_{I}$ and $\rho_{Q}$ are usually assumed as identical, given by normalizing the value of 
$R_{I}(m)$ over $R_{I}(0)$. Similarly, the cross-correlation coefficient is $\hat{\rho}_{I Q}(m)=R_{I Q}(m) /\left(\hat{\sigma}_{I} \hat{\sigma}_{Q}\right)$, where $\hat{\sigma}_{I}$ and $\hat{\sigma}_{Q}$ are the estimated standard deviations of $x_{I}$ and $x_{Q}$, respectively.

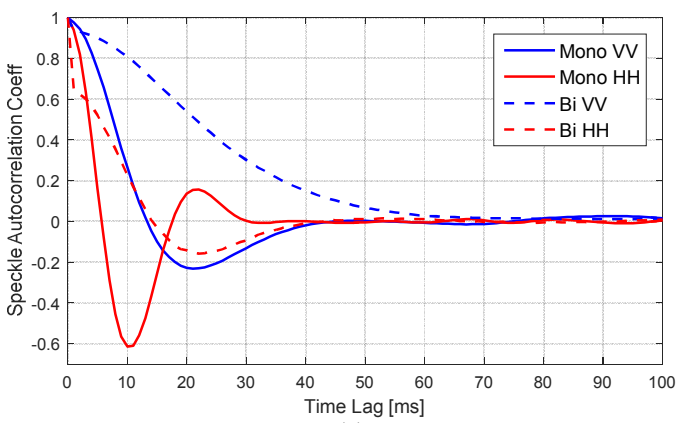

(a)

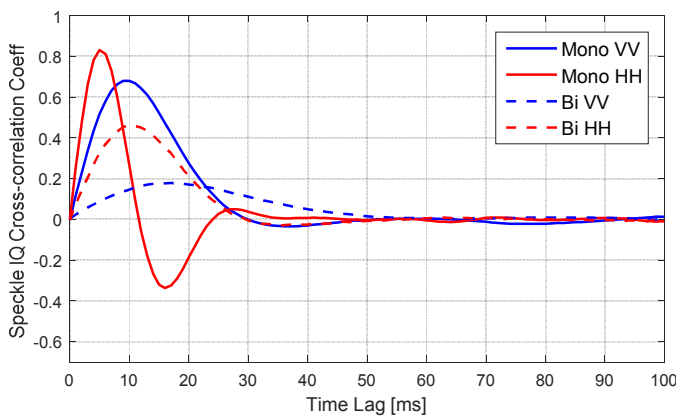

(b)

Fig 3: Autocorrelation of the in-phase speckle component $\rho_{I}$ (a) and crosscorrelation between the I and Q speckle components, $\rho_{I Q}(\mathrm{~b})$.Co-polarized data, $\beta=75^{\circ}$

Fig. 3 shows the behaviour of $\rho_{I}$ and $\rho_{I Q}$ as a function of the time-lag (in milliseconds), for the co-polarized data, collected at $\beta=75^{\circ}$. The bistatic VV data show the longest decorrelation time, whereas only the monostatic $\mathrm{HH}$ data show some periodicity, which is revealed by a small peak at about 22 seconds.

In Fig.4, we show the values of the speckle decorrelation time $\left(t_{I}\right)$. In general, bistatic data show higher values of the speckle decorrelation time, in particular for the vertically polarized data. In all the bistatic data, $t_{I}$ shows an increasing behaviour of as a function of the bistatic angle, whereas the monostatic speckle shows an almost constant behaviour, which is justified by the fact that monostatic signal are not affected by the changes in geometry. Typical values of the monostatic speckle decorrelation time are included between $15 \mathrm{~ms}$ and $20 \mathrm{~ms}$. For the bistatic data, $t_{I}$ is included between $60 \mathrm{~ms}$ and $95 \mathrm{~ms}$ (VV), $20 \mathrm{~ms}$ and $40 \mathrm{~ms}$ (HH), $30 \mathrm{~ms}$ and $100 \mathrm{~ms}$ (HV), $20 \mathrm{~ms}$ and $65 \mathrm{~ms}(\mathrm{VH})$.

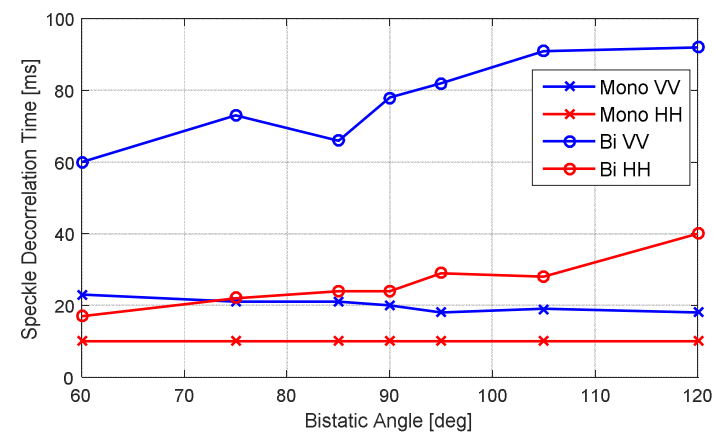

(a)

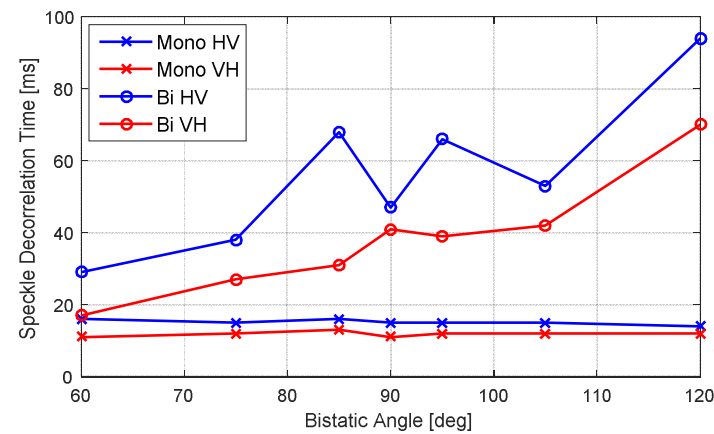

(b)

Fig.4: speckle decorrelation times of (a) Co-polarized data (b) Crosspolarized data

\section{Cross-Correlation}

The data that were simultaneously collected by the widely separated nodes of a multistatic radar system give the opportunity to evaluate the degree of correlation between the signals associated to two different channels. To this aim, we analyzed the cross-correlation between the estimated texture and speckle samples of the sea clutter data collected by the NetRAD. The cross-covariance function between the bistatic and monostatic textures, $R_{\tau}^{(M, B)}$, was calculated as

$$
R_{\tau}^{(M, B)}(l)=\frac{1}{N_{b}} \sum_{k=1}^{N_{b}-|l|}\left[\hat{\tau}_{M}(k)-\hat{\mu}_{\tau}^{(M)}\right]\left[\hat{\tau}_{B}(k+|l|)-\hat{\mu}_{\tau}^{(B)}\right]
$$

where $\hat{\tau}_{B}$ and $\hat{\tau}_{M}$ and represent the bistatic and monostatic texture, whose sample means are $\hat{\mu}_{\tau}^{(B)}$ and $\hat{\mu}_{\tau}^{(M)}$.The texture cross-correlation coefficient - $\hat{\rho}_{\tau}^{(M, B)}(l)$ - is obtained by normalizing $R_{\tau}^{(M, B)}(l)$ over the monostatic and bistatic texture standard deviations.

Fig. 5 shows the behaviour of $\hat{\rho}_{\tau}^{(M, B)}$ as a function of time-lag (measured in seconds), for the co-polarized data collected at a bistatic angle of 75 degrees. The values of $\hat{\rho}_{\tau}^{(M, B)}$ fluctuate within the interval $[-0.1,0.2]$ for the $\mathrm{HH}$ data and between 0.1 and 0.5 for VV data. The vertically-polarized data show a "more sinusoidal" behaviour of $\hat{\rho}_{\tau}^{(M, B)}$ with respect to the horizontal-polarized ones, with peaks occurring every 5-7 seconds. In order to quantify the degree of correlation between the monostatic and bistatic texture samples, we 
chose the maximum value of $\hat{\rho}_{\tau}^{(M, B)}$, which is plotted in Fig. 6 as a function of the bistatic angle. In general, the maximum texture cross-correlation coefficient fluctuates between 0.2 and 0.5 for co-polarized data, and between 0.7 and 0.05 for cross-polarized data.

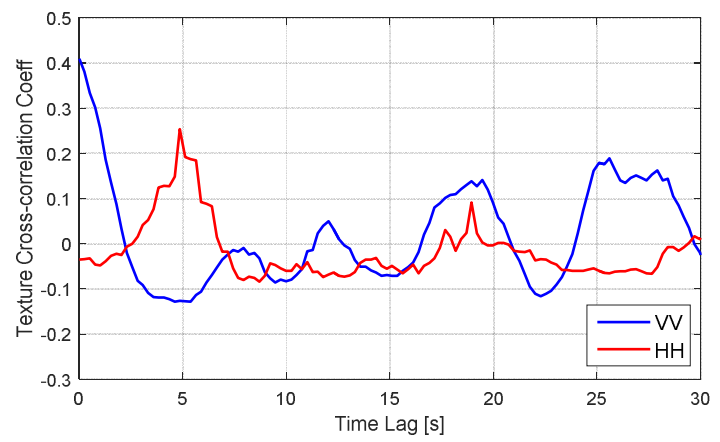

Fig.5: Monostatic-bistatic texture cross-correlation (Co-polarized data, $\beta=$ $\left.75^{\circ}\right)$

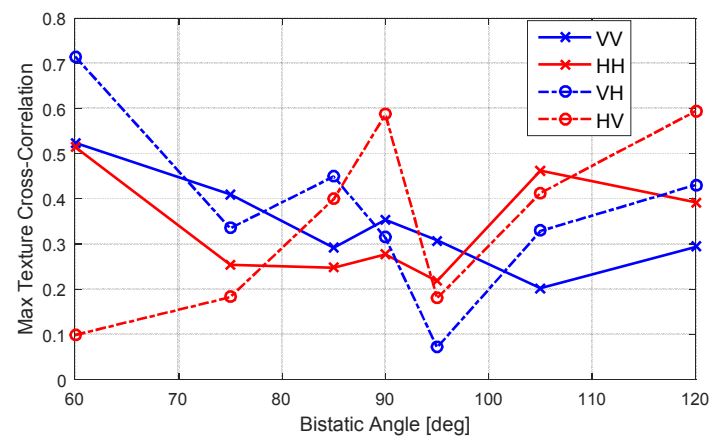

Fig.6 - Maximum value of the texture cross-correlation as a function of the bistatic angle

Concerning the analysis of the speckle, its in-phase component was only considered, as it is reasonable to assume that its quadrature component has similar properties. The cross-correlation coefficient between the bistatic and monostatic in-phase speckle samples was calculated as

$$
R_{I}^{(M, B)}(m)=\frac{1}{N_{p}} \sum_{k=1}^{N_{p}-|m|} \hat{x}_{I}^{(M)}(n) \hat{x}_{I}^{(B)}(n+|m|)
$$

where $\hat{x}_{I}^{(B)}$ and $\hat{x}_{I}^{(M)}$ are the bistatic and monostatic in-phase speckle estimates (obtained by normalizing the clutter samples over the texture estimates). The speckle crosscorrelation coefficient $\hat{\rho}_{I}^{(M, B)}(m)$ is obtained by normalizing $R_{I}^{(M, B)}(m)$ over the estimated standard deviations of the monostatic and bistatic in-phase components.

Fig.7 shows the behaviour of $\hat{\rho}_{I}^{(M, B)}$ as a function of time-lag (measured in milliseconds), for the data collected at a bistatic angle of $75^{\circ}$. The values of the cross-correlation coefficient are very small, fluctuating between -0.01 and 0.01 , meaning that the degree of correlation between the bistatic and monostatic speckle samples is almost negligible. The maximum values of $\hat{\rho}_{I}^{(M, B)}$ are plotted in Fig.8 as a function of the bistatic angle. The VV data show a higher degree of correlation between the monostatic and bistatic speckle samples, with a peak at $85^{\circ}-90^{\circ}$.

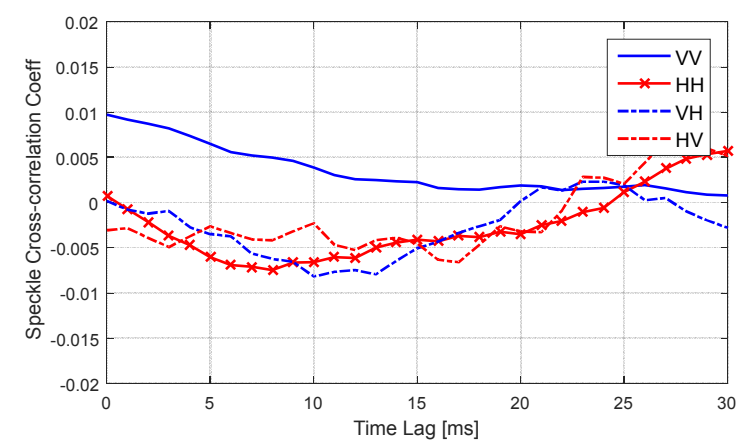

Fig 7 Monostatic-bistatic in-phase speckle cross correlation coefficient $(\beta=$ $\left.75^{\circ}\right)$

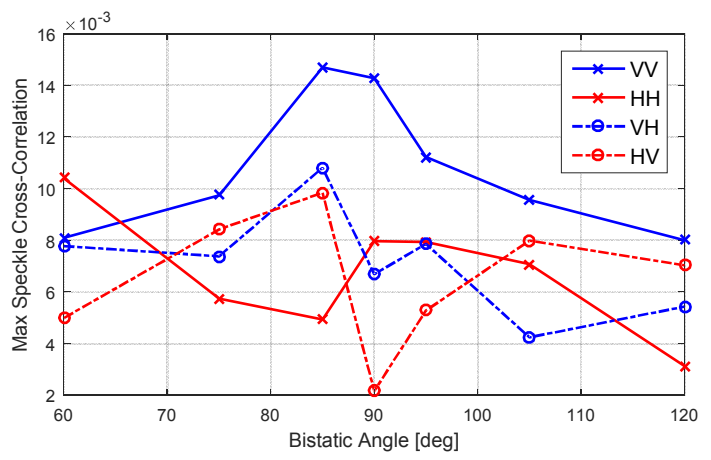

Fig 8 - Maximum value of the speckle cross-correlation coefficient as a function of the bistatic angle

\section{CONCLUSIONS}

This paper has shown a wide range of comparative results of both texture and speckle components as a function of bistatic angle and polarization. The effect of bistatic geometry on the speckle temporal correlation varied the monostatic results very little, while the bistatic values increased approximately linearly with increasing $\beta$. This was shown to be true for both the co and cross polarized datasets. The effect was also observed, to a lesser extent, for the cross polarized texture correlation. While the co-polarized texture was shown to peak at $90^{\circ}$ and $120^{\circ}$ for the majority of datasets.

Further analysis of how discrete spiking events observed both in the monostatic and bistatic channels evolve with time will be addressed in future works. As sea spikes are critical components of a sea clutter distribution their correlation across a multistatic radar sensor network are of great interest.

\section{ACKNOWLEDGEMENT}

The authors would like to thank Dr Waddah Al-Ashwal for generating these datasets, ONR-G, IET AF Harvey award and EPSRC that have made substantial contribution to equipment and post-doctoral funds. 


\section{REFERENCES}

[1] N. J. Willis and H. D. Griffiths, Advances on bistatic radar. .

[2] M. Inggs, H. Griffiths, F. Fioranelli, M. Ritchie, and K. Woodbridge, "Multistatic radar: System requirements and experimental validation," 2014 Int. Radar Conf. Radar 2014, pp. $1-6,2014$.

[3] A. Farina, F. Gini, M. V. Greco, and L. Verrazzani, "High resolution sea clutter data: statistical analysis of recorded live data," IEE Proc. - Radar, Sonar Navig., vol. 144, no. 3, p. 121, 1997.

[4] K.D. Ward, R. J. A Tough, , and S.Watts, Sea Clutter: Scattering, the $K$ Distribution and Radar Performance, London, United Kingdom: IET, 2006.

[5] L. Rosenberg, "The effect of temporal correlation with $\mathrm{K}$ and KK-distributed sea-clutter"2012 IEEE Radar Conference, Atlanta, GA, 2012, pp. 0303-0308.

[6] Chong C.Y.; Pascal, F.; Ovarlez, J.; Lesturgie, M., "MIMO Radar Detection in Non-Gaussian and Heterogeneous Clutter,"IEEE Journal of Selected Topics in Signal Processing, , vol.4, no.1, pp. 115,126 , Feb. 2010

[7] R. Palamà, M. Greco, and F. Gini, "Multistatic adaptive CFAR detection in non-Gaussian clutter," EURASIP J. Adv. Signal Process., pp. 1-17, 2016.

[8] Michels, J.H.; Varshney, P.; Weiner, D., "Multichannel signal detection involving temporal and cross-channel correlation," in, IEEE Transactions on Aerospace and Electronic Systems, 31, 3, pp866-880, Jul 1995

[9] W. A. M. Al-Ashwal, "Measurement and Modelling of Bistatic Sea Clutter,", PhD dissertation, University College London, 2011.

[10] J. S. Sandenbergh, M. R. Inggs, and W. A. Al-Ashwal, "Evaluation of coherent netted radar carrier stability while synchronised with GPS-disciplined oscillators," IEEE Radar Conf. - Proc., vol. 1, no. 1, pp. 1100-1105, 2011.

[11] R. Palamà, M. S. Greco, P. Stinco, and F. Gini, "Statistical Analysis of Bistatic and Monostatic Sea Clutter," IEEE Trans. Aerosp. Electron. Syst., vol. 51, no. 4, pp. 3036-3054, 2015.

[12] F. Fioranelli, M. Ritchie, H. Griffiths, M. Inggs, and S. Sandenbergh, "Analysis of polarimetric bistatic sea clutter using the NetRAD radar system," IET Radar, Sonar Navig., p. (doi:10.1049/iet-rsn.2015.0416) (Early Online Publ, 2016. 